\title{
Quantum and transport conductivities in monolayer graphene
}

\author{
H. M. Dong, ${ }^{1}$ W. Xu, ${ }^{1,2, *}$ Z. Zeng, ${ }^{1}$ T. C. Lu ${ }^{3}$ and F. M. Peeters ${ }^{4, \dagger}$ \\ ${ }^{1}$ Institute of Solid State Physics, Chinese Academy of Sciences, Hefei 230031, People's Republic of China \\ ${ }^{2}$ Department of Theoretical Physics, Research School of Physical Sciences and Engineering, Australian National University, \\ Canberra ACT 0200, Australia \\ ${ }^{3}$ Department of Physics, Sichuan University, Chengdu 610064, People's Republic of China \\ ${ }^{4}$ Department of Physics, University of Antwerp, Groenenborgerlaan 171, B-2020 Antwerpen, Belgium
}

(Received 18 February 2008; published 2 June 2008)

\begin{abstract}
We present a detailed theoretical study of the electronic transport properties of monolayer graphene. The quantum and transport conductivities are calculated on the basis of the usual momentum-balance equation derived from a semiclassical Boltzmann equation. We investigate carrier-impurity scattering in a massless Dirac quasiparticle system. The carrier interactions with remote and background impurities are considered, and the carrier-carrier screening is included within the random phase approximation. The dependence of the conductivities on temperature is also examined. Moreover, a very simple analytical formula is proposed such that only one fitting parameter is needed in order to make a quantitative comparison with the experimental results.
\end{abstract}

DOI: 10.1103/PhysRevB.77.235402

PACS number(s): 73.63.Hs, 72.15.Lh, 71.70.Ej

\section{INTRODUCTION}

The realization of electronic devices based on a single sheet of graphite (or graphene) is an important scientific breakthrough. ${ }^{1-3}$ From a fundamental physics point of view, graphene provides us with an opportunity to study massless and gapless Dirac quasiparticles that differ essentially from the conventional Schrödinger particles. ${ }^{4}$ On the other hand, owing to its unique electronic band structure and corresponding quasirelativistic features, graphene has been proposed as a new electronic system for advanced devices such as graphene $p-n$ junctions,${ }^{5} p-n-p$ junctions, ${ }^{6}$ transistors, ${ }^{7}$ etc. As a result, the study of electronic transport of Dirac quasiparticles in graphene has become an important research topic in condensed matter physics and nanoelectronics. ${ }^{4}$

At present, the often used graphene device is based on a graphene sheet that is placed on top of an oxidized silicon wafer. $^{1-3}$ One of the major advantages of such a device is that the density of conducting carriers in the graphene layer can be controlled very effectively through a gate voltage applied perpendicular to the graphene sheet. It is found experimentally that in typical graphene on a $\mathrm{SiO}_{2}$ wafer, the carrier density increases almost linearly with the gate voltage, with over a wide range of gate voltage away from the charge neutrality (Dirac) point. ${ }^{1,7}$ In this linear density-voltage regime, the conductivity in a single layer graphene increases almost linearly with the gate voltage. ${ }^{8}$ Consequently, the carrier mobility is constant and independent of the carrier density. ${ }^{9}$

In conjunction with intensive experimental activities in the study of the electronic transport properties in graphene devices, a number of theoretical studies have been carried out to calculate the transport conductivity induced by impurity $^{4,9,10}$ and phonon ${ }^{11,12}$ scattering in graphene. Similar to the investigation of conventional electron gas systems, the theoretical approach based on the Boltzmann equation ${ }^{10,11}$ has been popularly used to calculate the conductivity or resistivity in graphene. It should be noted that the relaxation time $\langle\tau\rangle$ obtained by Hwang et al. ${ }^{10}$ and $\langle 1 / \tau\rangle$ by Vasko and Ryzhii ${ }^{11}$ are determined simply by the energy average over the scattering rate from Fermi's golden rule. Similar approach has also been used by Nomura and MacDonald ${ }^{9}$ to calculate the relaxation time as it is limited by impurity scattering at zero magnetic field. In particular, in Refs. 9, $\langle\tau\rangle$ $\sim \int d E E \tau(E)[-d f(E) / d E]$, and $11,\langle 1 / \tau\rangle \sim \int d E E[1 / \tau(E)]$ $\times[-d f(E) / d E]$, were used to evaluate the average relaxation time. We know that these are correct formulas for an electron gas system with a parabolic dispersion relation $E \sim k^{2}$. For the case of graphene that has a linear dispersion $E \sim k$, one needs to check if these formulas still hold. As pointed out by Hwang et al., ${ }^{10}$ the carrier-carrier screening plays an important role in affecting the effective carrier-impurity scattering and the low-temperature conductivity in graphene. In Ref. 9, the inverse screening length $K_{s}$ at long-wavelength regime (i.e., $q \ll 1$ ) was used to calculate the conductivity. When $q$ $\ll 1, K_{s}$ is proportional to the Fermi wave vector so that the conductivity is definitely linear in the electron density. A question one would like to ask is that if we do not take the small $q$ approximation for the screening length, which, in general, is a functional form of the electron density, how would the transport conductivity be modified?

Motivated by the experimental and theoretical works mentioned above, in this paper we would like to contribute a more detailed study to improve the theoretical investigation of the transport properties in graphene. We intend to develop a simple and transparent theory to calculate the quantum and transport conductivities in graphene. Our model is based on the balance-equation approach using the semiclassical Boltzmann equation. Thus, the momentum relaxation time can be obtained more straightforwardly and more accurately. We consider the presence of both remote and background impurities, and evaluate the strength of these scattering mechanisms. In the study, we also examine the effect of carrierimpurity scattering on the quantum conductivity in graphene. In contrast to conventional transport conductivity that is obtained simply from $I-V$ measurement, the quantum conductivity is measured from the amplitudes of the Shubnikov-de 
Haas (SdH) oscillations via the famous Dingle plot. ${ }^{13}$ Thus, the quantum conductivity can reflect the consequences of small-angle scattering in an electronic device. Because the $\mathrm{SdH}$ oscillations have been well observed in graphene even at relatively high temperatures ${ }^{1,2}$ the quantum conductivity in graphene can be obtained easily from the experimental data. In our calculations, we do not take the small $q$ approximation for the screening length. Therefore, the effect of carriercarrier screening is more correctly included in this study as compared to previous research. Furthermore, we would like to find out a simple theoretical way to study the dependence of the conductivity induced by the impurity scattering on temperature in graphene devices. From a practical point of view, we would also like to reach a simple analytical expression in order to compare with the experimental data more easily.

The paper is organized as follows. The single-particle aspects and the carrier-carrier screening length induced by many-body interaction in graphene are briefly examined in Secs. II and III. In Secs. IV and V, we study the carrierimpurity scattering and the quantum and transport conductivities in graphene analytically. The numerical results are presented and discussed in Sec. VI, and the concluding remarks are summarized in Sec. VII.

\section{SINGLE-PARTICLE ASPECTS}

In our model we consider a graphene sheet in the $x y$ plane placed on top of a $\mathrm{SiO}_{2}$ wafer. Using the effective-mass approximation, a carrier (electron or hole) in a monolayer graphene can be described by Weyl's equation for a massless neutrino. ${ }^{14}$ The single-particle Hamiltonian, describing a carrier in the $\pi$ bands near a $K$ point in graphene, can be obtained from, e.g., the $\mathbf{k} \cdot \mathbf{p}$ approach, ${ }^{14}$ which reads

$$
H_{0}=\gamma\left[\begin{array}{cc}
0 & k_{x}-i k_{y} \\
k_{x}+i k_{y} & 0
\end{array}\right]
$$

where $\gamma=\hbar v_{F}$ is the band parameter with $v_{F} \simeq 10^{8} \mathrm{~cm} / \mathrm{s}$ being the Fermi velocity of a Dirac particle and $k_{x}=-i \partial / \partial x$ is the wave vector operator along the $x$ direction. The corresponding eigenvalue and eigenfunction are, respectively

$$
E_{\lambda}(\mathbf{k})=\lambda \gamma|\mathbf{k}|=\lambda \gamma k
$$

and

$$
\psi_{\lambda \mathbf{k}}(\mathbf{r})=\langle\mathbf{r} \mid \mathbf{k}, \lambda\rangle=2^{-1 / 2}\left[1, \lambda e^{i \phi}\right] e^{i \mathbf{k} \cdot \mathbf{r}}
$$

in the form of a row matrix. Here, $\mathbf{k}=\left(k_{x}, k_{y}\right)$ is the wave vector for a carrier. $k=\sqrt{k_{x}^{2}+k_{y}^{2}}, \mathbf{r}=(x, y)$, and $\lambda=+1$ is for an electron, and $\lambda=-1$ is for a hole. $\phi$ is the angle between $\mathbf{k}$ and the $x$ direction.

The free-particle Green's function for a carrier is

$$
G_{\lambda \mathbf{k}}(E)=\left[E-E_{\lambda}(\mathbf{k})+i \delta\right]^{-1},
$$

with $E$ being the carrier energy. Thus, the density of states (DOS) for the system is determined by the imaginary part of the Green's function, which reads

$$
D_{\lambda}(E)=g_{s} g_{v} \sum_{\mathbf{k}} \delta\left[E-E_{\lambda}(\mathbf{k})\right]=\frac{g_{s} g_{v}|E|}{2 \pi \gamma^{2}} \Theta(\lambda E),
$$

where $g_{s}=2$ and $g_{v}=2$ count, respectively, for the spin and valley degeneracies. With the carrier DOS, the Fermi energy $E_{F}$ (or chemical potential $\mu^{*}$ ) of the system can be determined by the condition of carrier number conservation. Using the Fermi-Dirac function as statistic energy distribution for a carrier, we have

$$
n_{\lambda}=\frac{2}{\pi}\left(\frac{k_{B} T}{\gamma}\right)^{2} \int_{0}^{\infty} \frac{d x x}{\beta^{\lambda} e^{x}+1},
$$

where $n_{+}\left(n_{-}\right)$is the electron (hole) density and $\beta=e^{-\mu^{*} / k_{B} T}$. In particular, for a high carrier-density sample at low temperature, so that $E_{F} \gg k_{B} T$, we have

$$
\mu^{*} \simeq E_{F}-\left(\pi k_{B} T\right)^{2} /\left(6 E_{F}\right)
$$

with

$$
E_{F}=\lambda \gamma \sqrt{\pi n_{\lambda}}
$$

being the Fermi energy at $T \rightarrow 0$, and the corresponding Fermi wave vector is

$$
k_{F}=\sqrt{\pi n_{\lambda}} .
$$

\section{SCREENING LENGTH IN THE RANDOM PHASE APPROXIMATION}

With the single-particle carrier wave function, we can calculate the electrostatic energy induced by the bare carriercarrier $(c-c)$ interaction

$$
\begin{aligned}
V_{\lambda_{1}^{\prime} \lambda_{1} \lambda_{2}^{\prime} \lambda_{2}}= & S_{\lambda_{1}^{\prime} \lambda_{1} \lambda_{2}^{\prime} \lambda_{2}} \int d^{2} \mathbf{r}_{1} d^{2} \mathbf{r}_{2} \psi_{\lambda_{1}^{\prime} \mathbf{k}_{1}^{\prime}}^{*}\left(\mathbf{r}_{1}\right) \psi_{\lambda_{1} \mathbf{k}_{1}}\left(\mathbf{r}_{1}\right) \\
& \times V\left(\mathbf{r}_{1}-\mathbf{r}_{2}\right) \psi_{\lambda_{2}^{\prime} \mathbf{k}_{2}^{\prime}}^{*}\left(\mathbf{r}_{2}\right) \psi_{\lambda_{2} \mathbf{k}_{2}}\left(\mathbf{r}_{2}\right),
\end{aligned}
$$

where $V(\mathbf{r})=e^{2} / \kappa|\mathbf{r}|$ is the Coulomb potential with $\kappa$ being the static dielectric constant of the electronic system, and $S_{\lambda_{1} \lambda_{2} \lambda_{3} \lambda_{4}}=-i^{\left(\lambda_{1}+\lambda_{2}+\lambda_{3}+\lambda_{4}\right) / 2}= \pm 1$ or $\pm i$ is a sign function related to the charge sign of the carriers involved in the scattering process. Due to momentum conservation for a $c-c$ scattering event, ${ }^{15}$ we arrive at the bare $c-c$ interaction matrix element

$$
V_{\alpha \beta}=S_{\alpha \beta} V_{q} G_{\alpha \beta}(\mathbf{k}, \mathbf{q}),
$$

where we have defined $\alpha=\left(\lambda^{\prime} \lambda\right)$ for intra- (i.e., $\lambda^{\prime}=\lambda$ ) and interband (i.e., $\left.\lambda^{\prime} \neq \lambda\right)$ transition, $\mathbf{q}=\left(q_{x}, q_{y}\right)$ is the change of the carrier wave vector during the $c-c$ scattering event, $V_{q}$ $=2 \pi e^{2} / \kappa q$, and

$$
G_{\alpha \beta}(\mathbf{k}, \mathbf{q})=\frac{1+\alpha A_{\mathbf{k q}}}{2} \delta_{\alpha, \beta}+i \frac{\alpha B_{\mathbf{k q}}}{2}\left(1-\delta_{\alpha, \beta}\right),
$$

with $A_{\mathbf{k q}}=(k+q \cos \theta) /|\mathbf{k}+\mathbf{q}|, B_{\mathbf{k q}}=(q \sin \theta) /|\mathbf{k}+\mathbf{q}|$, and $\theta$ being the angle between $\mathbf{k}$ and $\mathbf{q}$.

Within the random phase approximation (RPA), the effective $c-c$ interaction can be calculated through 


$$
V_{\alpha \beta}^{\mathrm{eff}}=\epsilon_{\alpha \beta}^{-1} V_{\alpha \beta}
$$

where

$$
\epsilon(\Omega, q)=\left[\begin{array}{cccc}
1+a_{1} & 0 & 0 & -a_{4} \\
0 & 1-a_{2} & -a_{3} & 0 \\
0 & -a_{2} & 1-a_{3} & 0 \\
-a_{1} & 0 & 0 & 1+a_{4}
\end{array}\right]
$$

is the dynamical dielectric function matrix with $\Omega$ being the excitation frequency. Here the indexes $\alpha=\left(\lambda^{\prime} \lambda\right)=(++)=1$, $(+-)=2,(-+)=3$, and $(--)=4$ are defined according to the different transition channels. Furthermore, $a_{j}=-V_{q} \Pi_{j}(\Omega, q)$ where

$$
\begin{aligned}
\Pi_{\lambda^{\prime} \lambda}(\Omega, q)= & \frac{g_{s} g_{v}}{2} \sum_{\mathbf{k}}\left(1+\lambda^{\prime} \lambda A_{\mathbf{k q}}\right) \\
& \times \frac{f\left[E_{\lambda^{\prime}}(\mathbf{k}+\mathbf{q})\right]-f\left[E_{\lambda}(\mathbf{k})\right]}{\hbar \Omega+E_{\lambda^{\prime}}(\mathbf{k}+\mathbf{q})-E_{\lambda}(\mathbf{k})+i \delta}
\end{aligned}
$$

is the pair bubble or density-density correlation function in the absence of $c-c$ screening. Here we utilize a matrix to present the dielectric function, which differs slightly from previous presentations. ${ }^{10}$ The inverse dielectric function matrix is

$$
\boldsymbol{\epsilon}^{-1}(\Omega, q)=\left[\begin{array}{cccc}
1-a_{1}^{*} & 0 & 0 & a_{4}^{*} \\
0 & 1+a_{2}^{*} & a_{3}^{*} & 0 \\
0 & a_{2}^{*} & 1+a_{3}^{*} & 0 \\
a_{1}^{*} & 0 & 0 & 1-a_{4}^{*}
\end{array}\right]
$$

with $a_{1}^{*}=a_{1} /\left(1+a_{1}+a_{4}\right), a_{2}^{*}=a_{2} /\left(1-a_{2}-a_{3}\right), a_{3}^{*}=a_{3} /\left(1-a_{2}\right.$ $\left.-a_{3}\right)$, and $a_{4}^{*}=a_{4} /\left(1+a_{1}+a_{4}\right)$. The inverse dielectric function matrix can be used to calculate the effective interaction matrix element for carrier coupling with scattering centers.

In particular, for the case where only the electron states are occupied and only the e-e interaction (i.e., $\alpha=1$ ) is present, we have

$$
\operatorname{Re} \Pi_{1}(q)=\frac{-1}{\pi^{2} \gamma} \int_{0}^{\infty} d k f(\gamma k)[\pi+I(k, q)-I I(k, q)],
$$

with

$$
I(k, q)=\frac{2 b[q+4 k \Theta(q-k)]}{a q} K\left(\frac{\sqrt{4 k q}}{a}\right)
$$

and

$$
I I(k, q)=\frac{8 b(a+k)}{q(a+b)} \Pi\left(\frac{\pi}{2}, \frac{a-b}{a+b} \cdot \frac{k+b}{k-b}, \frac{a-b}{a+b}\right),
$$

where $a=k+q, b=|k-q|$, and $K(x)$, and $\Pi(\phi, n, x)$ are, respectively, the complete elliptic integrals of the first and third kind. Thus, the real part of the static RPA dielectric function induced by electron-electron interaction takes the form $\operatorname{Re} \epsilon_{1}(q)=\operatorname{Re} \epsilon_{1}(0, q)=1+K_{s} / q$, with

$$
K_{s}=\frac{2 e^{2}}{\pi \kappa \gamma} \int_{0}^{\infty} d k f(\gamma k)[\pi+I(k, q)-I I(k, q)]
$$

being the inverse RPA screening length. For low temperature (i.e., $T \rightarrow 0$ ),

$$
K_{s}=\frac{q_{s}}{2}+\frac{q_{s}}{2 \pi k_{F}} \int_{0}^{k_{F}} d k[I(k, q)-I I(k, q)],
$$

with $q_{s}=4 e^{2} k_{F} / \kappa \gamma$ being the Thomas-Fermi wave vector for graphene. In the long-wavelength limit (i.e., $q \rightarrow 0$ ),

$$
K_{s} \simeq q_{s}\left(1-\pi q / 8 k_{F}\right),
$$

a result obtained previously in Ref. 9.

\section{CARRIER-IMPURITY SCATTERING}

At relatively low-temperatures, the carrier-impurity $(c-i)$ scattering is the principle channel for relaxation of excited carriers in electronic systems. For the case where the $c-i$ scattering is achieved through the Coulomb potential induced by charged impurities that are three-dimensional-like, the interaction Hamiltonian is

$$
H_{c-i}=\frac{Z e^{2}}{\kappa_{I}} \frac{1}{\left|\mathbf{R}-\mathbf{R}_{a}\right|},
$$

where $\mathbf{R}=(\mathbf{r}, 0)$ is the coordinates of a carrier in the graphene layer, the impurity with a charge number $Z$ is located at $\mathbf{R}_{a}=\left(\mathbf{r}_{a}, z_{a}\right)$, and $\kappa_{I}$ is the static dielectric constant of the medium that contains the impurities. After assuming that the system can be separated into the carriers of interest $|\mathbf{k}, \lambda\rangle$ and the rest of impurities $|I\rangle$, namely $|\mathbf{k}, \lambda ; I\rangle=|\mathbf{k}, \lambda\rangle|I\rangle$, the matrix element for $c-i$ interaction is obtained, in the absence of carrier-carrier $(c-c)$ screening, as

$$
\begin{aligned}
U_{\lambda^{\prime} \lambda}\left(q, \mathbf{R}_{a}\right) & =\left\langle\mathbf{k}^{\prime}, \lambda^{\prime} ; I^{\prime}\left|H_{c-i}\right| \mathbf{k}, \lambda ; I\right\rangle \\
& =\frac{\pi Z e^{2}}{\kappa_{I} q} e^{-q\left|z_{a}\right|} \sqrt{n_{i}\left(z_{a}\right)}\left(1+\lambda \lambda^{\prime} e^{i \theta}\right) e^{-i \mathbf{q} \cdot \mathbf{r}_{a}} \delta_{\mathbf{k}, \mathbf{k}+\mathbf{q}},
\end{aligned}
$$

where $\left\langle I^{\prime} \mid I\right\rangle=\sqrt{n_{i}\left(z_{a}\right)}$ with $n_{i}(z)$ being the impurity distribution along the $z$ direction, $\theta$ is the angle between $\mathbf{k}^{\prime}$ and $\mathbf{k}$, and $\mathbf{q}=\left(q_{x}, q_{y}\right)$ is the change of the carrier wave vector during a scattering event. Here we have assumed that the impurities are distributed uniformly along the $x y$ plane. Using Fermi's golden rule, the electronic transition rate for scattering of a carrier from a state $|\mathbf{k}, \lambda\rangle$ to a state $\left|\mathbf{k}^{\prime}, \lambda^{\prime}\right\rangle$ due to $c-i$ interaction is obtained as

$$
W_{\lambda^{\prime} \lambda}\left(\mathbf{k}^{\prime}, \mathbf{k}\right)=\frac{2 \pi}{\hbar}\left|U_{\lambda^{\prime} \lambda}(q, \theta)\right|^{2} \delta_{\mathbf{k}^{\prime}, \mathbf{k}+\mathbf{q}} \delta\left[E_{\lambda^{\prime}}\left(\mathbf{k}^{\prime}\right)-E_{\lambda}(\mathbf{k})\right],
$$

where $\left|U_{\lambda^{\prime} \lambda}(q, \theta)\right|^{2}=\left(2 \pi Z e^{2} / \kappa_{I} q\right)^{2}\left(1+\lambda^{\prime} \lambda \cos \theta\right) / 2 \int d z n_{i}(z)$ $\times e^{-2 q|z|}$, in which we have integrated over the contributions from all impurities along the $z$ direction.

In the presence of $c-c$ screening, the effective $c-i$ scattering matrix element can be obtained through 


$$
U_{j}^{\mathrm{eff}}(q, \theta)=\sum_{j^{\prime}} \epsilon_{j j^{\prime}}^{-1} U_{j^{\prime}}(q, \theta),
$$

which gives, after using Eq. (15),

$$
\begin{aligned}
& U_{1}^{\mathrm{eff}}(q, \theta)=\frac{\left(1+b_{4}\right) U_{1}(q, \theta)+b_{4} U_{4}(q, \theta)}{1+b_{1}+b_{4}}, \\
& U_{2}^{\mathrm{eff}}(q, \theta)=\frac{\left(1-b_{3}\right) U_{2}(q, \theta)+b_{3} U_{3}(q, \theta)}{1-b_{2}-b_{3}}, \\
& U_{3}^{\mathrm{eff}}(q, \theta)=\frac{b_{2} U_{2}(q, \theta)+\left(1+b_{2}\right) U_{3}(q, \theta)}{1-b_{2}-b_{3}},
\end{aligned}
$$

and

$$
U_{4}^{\mathrm{eff}}(q, \theta)=\frac{b_{1} U_{1}(q, \theta)+\left(1+b_{1}\right) U_{4}(q, \theta)}{1+b_{1}+b_{4}},
$$

where again $\left(\lambda^{\prime} \lambda\right)=1=(++),=2=(+-),=3=(-+),=4=(--)$, and $b_{j}=\lim _{\Omega \rightarrow 0} a_{j}$ is the static dielectric matrix element with $a_{j}$ given in Eq. (13). In particular, for the case where only the electron states are occupied, we have $b_{4}=0$ and $U_{1}^{\text {eff }}(q, \theta)$ $=U_{1}(q, \theta) /\left(1+b_{1}\right)$.

\section{QUANTUM AND TRANSPORT CONDUCTIVITIES}

In this work, we employ the Boltzmann equation as the governing transport equation to study the response of the carriers in graphene to the applied dc driving field. The semiclassical Boltzmann equation in the presence of an external electrical field $F_{x}$ applied along the $x$ direction is

$$
\begin{aligned}
-\frac{e F_{x}}{\hbar} \frac{\partial f_{\lambda}(\mathbf{k})}{\partial k_{x}}= & g_{s} g_{v} \sum_{\mathbf{k}^{\prime}, \lambda^{\prime}}\left[f_{\lambda^{\prime}}\left(\mathbf{k}^{\prime}\right) W_{\lambda \lambda^{\prime}}\left(\mathbf{k}, \mathbf{k}^{\prime}\right)\right. \\
& \left.-f_{\lambda}(\mathbf{k}) W_{\lambda^{\prime} \lambda}\left(\mathbf{k}^{\prime}, \mathbf{k}\right)\right]
\end{aligned}
$$

where $f_{\lambda}(\mathbf{k})$ is the momentum-distribution function for a carrier in a state $|\mathbf{k}, \lambda\rangle$. It is known that there is no simple and analytical solution to Eq. (24) with the electronic transition rate given by Eq. (22). In this study we utilize the usual balance-equation approach to solve the problem. The balance-equation approach is a powerful tool in investigating transport properties of the electron gas systems. We demonstrated previously that the results obtained from the momentum- and energy-balance equations derived on the basis of the Boltzmann equation agree very well with those obtained from the Monte Carlo simulations for conventional two-dimensional electron gases (2DEGs). ${ }^{16}$ The results obtained from this approach also agree well with experimental data for GaAs-based 2DEGs driven by intense terahertz laser fields ${ }^{17}$ and for InGaAs-based Rashba spintronic systems. ${ }^{18}$ In this paper, we generalize this well developed theoretical approach to the case for the Dirac quasiparticle in graphene. For the first moment, the momentum-balance (or forcebalance) equation ${ }^{18}$ can be derived by multiplying $g_{s} g_{v} \sum_{\mathbf{k}, \lambda} k_{x}$ on both sides of the Boltzmann equation. Using the following properties:

$$
g_{s} g_{v} \sum_{\mathbf{k}, \lambda} f_{\lambda}(\mathbf{k})=N_{0}=n_{e}+n_{h}
$$

which is the total conducting-carrier density in the system, and

$$
g_{s} g_{v} \sum_{k, \lambda} k_{x} \frac{\partial f_{\lambda}(\mathbf{k})}{\partial k_{x}}=-N_{0}
$$

we obtain

$$
\frac{e F_{x}}{\hbar} N_{0}=16 \sum_{\mathbf{k}^{\prime}, \mathbf{k} \lambda^{\prime}, \lambda} \sum_{x}\left(k_{x}^{\prime}-k_{x}\right) f_{\lambda}(\mathbf{k}) W_{\lambda^{\prime} \lambda}\left(\mathbf{k}^{\prime}, \mathbf{k}\right) .
$$

In this study, we consider the case of linear response for the carriers in graphene to the applied dc driving field. For linear response, we do not need to solve the energy-balance equation, which is the second moment of the balance-equation approach. One of the advantages of the balance-equation approach is that we can circumvent the difficulties of solving the Boltzmann equation directly by using a specific form of the distribution function to calculate the interested physical properties. In this study, we assume that the momentum distribution of a carrier can be described by a statistical energy distribution function through $f_{\lambda}(\mathbf{k}) \simeq f\left[E_{\lambda}(\mathbf{k}-\nu \mathbf{v})\right]$, in which the momentum is shifted due to the presence of the driving field $F_{x}$; where $\mathbf{v}=\left(v_{x}, 0,0\right)$ is the average drift velocity of the carriers and $\nu$ is a coefficient that we have to determine such that $\nu v_{x}$ is the carrier momentum in graphene. We are intended in a situation where the applied dc electric field is so weak that $\nu v_{x} \ll k_{x}$ and, consequently, we have

$$
f_{\lambda}(\mathbf{k}) \simeq f\left(E_{\lambda}(k)\right)-\left.v_{x} \frac{\lambda \nu \gamma k_{x}}{k} f^{\prime}(X)\right|_{X=E_{\lambda}(\mathbf{k})},
$$

with $f^{\prime}(x)=d f(x) / d x$. Thus, the momentum-balance equation results in a linear response and

$$
\frac{1}{\mu_{t}}=\left.\frac{16 \hbar \nu \gamma}{e N_{0}} \sum_{\mathbf{k}^{\prime}, \lambda^{\prime}, \mathbf{k}, \lambda} \lambda\left(k_{x}^{\prime}-k_{x}\right) \frac{k_{x}}{k} W_{\lambda^{\prime} \lambda}\left(\mathbf{k}^{\prime}, \mathbf{k}\right) f^{\prime}(X)\right|_{X=E_{\lambda}(\mathbf{k})},
$$

where $\mu_{t}=-v_{x} / F_{x}$ is the transport mobility of the carriers. Noting that the transport mobility in graphene can also be defined as $\mu=e \tau_{t} / \hbar \nu$, the transport lifetime (or momentum relaxation time) can then be calculated through

$$
\frac{1}{\tau_{t}}=\left.\frac{16 \gamma}{N_{0}} \sum_{\mathbf{k}^{\prime}, \lambda^{\prime}, \mathbf{k}, \lambda} \lambda\left(k_{x}^{\prime}-k_{x}\right) \frac{k_{x}}{k} W_{\lambda^{\prime} \lambda}\left(\mathbf{k}^{\prime}, \mathbf{k}\right) f^{\prime}(X)\right|_{X=E_{\lambda}(\mathbf{k})},
$$

which reads, for $c-i$ scattering,

$$
\begin{aligned}
\frac{1}{\tau_{t}}= & \frac{-2}{\hbar \pi^{2} N_{0}} \sum_{\lambda} \lambda \int_{0}^{2 \pi} d \theta(1-\cos \theta) \\
& \times\left.\int_{0}^{\infty} d k k^{3}\left|U_{\lambda \lambda}^{\mathrm{eff}}(q, \theta)\right|^{2} f^{\prime}(X)\right|_{X=E_{\lambda}(k)},
\end{aligned}
$$

where $q=k \sqrt{2(1-\cos \theta)}$. By considering the fact that the 
quantum lifetime $\tau_{q}$ is induced by small-angle scattering events, we have

$$
\frac{1}{\tau_{q}}=\left.\frac{-2}{\hbar \pi^{2} N_{0}} \sum_{\lambda} \lambda \int_{0}^{2 \pi} d \theta \int_{0}^{\infty} d k k^{3}\left|U_{\lambda \lambda}^{\mathrm{eff}}(q, \theta)\right|^{2} f^{\prime}(X)\right|_{X=E_{\lambda}(k)} .
$$

These results suggest that for the case of carrier-impurity scattering, which is elastic, the momentum and energy conservation laws require that the interband scattering channel (i.e., $\lambda^{\prime} \neq \lambda$ ) is not allowed in graphene. Therefore, from now on we consider the conducting carriers in graphene to be electrons so that the Fermi energy of the system $E_{F}>0$. As a result, $f_{-}^{\prime}(x)=0$ for holes because all of the hole states are occupied by electrons, and $\left.f_{+}^{\prime}(x)\right|_{x=\gamma k}=f^{\prime}(\gamma k)$ $=d f(x) /\left.d x\right|_{x=\gamma k}$ for electrons. Thus, we obtain

$$
\begin{aligned}
{\left[\frac{1}{\tau_{t}}, \frac{1}{\tau_{q}}\right]=} & \frac{-2}{\pi^{2} \hbar n_{e}} \int_{0}^{\infty} d k k^{3} f^{\prime}(\gamma k) \int_{0}^{\pi} d \theta\left|U_{q}\right|^{2} \\
& \times\left[1-\cos ^{2} \theta, 1+\cos \theta\right],
\end{aligned}
$$

where $n_{e}$ is the electron density, $q=k \sqrt{2(1-\cos \theta)}$, and $\left|U_{q}\right|^{2}=\left[2 \pi Z e^{2} / \kappa\left(q+K_{s}\right)\right]^{2} \int d z n_{i}(z) e^{-2 q|z|}$ with $K_{s}$ being the inverse screening length given by Eq. (18).

Using the definition for the conductivity of graphene $\sigma$ $=\left(e^{2} / h\right)\left(2 E_{F} \tau / \hbar\right)$, which comes from the massless chiral Dirac spectrum, ${ }^{9,10}$ we obtain for the finite-temperature inverse transport and quantum conductivities,

$$
\frac{1}{\sigma_{l}(T)}=-\frac{1}{E_{F}^{3}} \int_{0}^{\infty} d E \frac{E^{3}}{\sigma_{l}(E, 0)} \frac{d f(E)}{d E},
$$

where $E=\gamma k$, and

$$
\left[\frac{\sigma_{0}}{\sigma_{t}(E, 0)}, \frac{\sigma_{0}}{\sigma_{q}(E, 0)}\right]=\frac{1}{\pi \gamma^{2}} \int_{0}^{\pi} d \theta\left|U_{q}\right|^{2}\left[A_{\theta}^{t}, A_{\theta}^{q}\right]
$$

with $\sigma_{0}=e^{2} / h, \quad A_{\theta}^{t}=1-\cos ^{2} \theta, \quad A_{\theta}^{q}=1+\cos \theta, \quad$ and $q$ $=(E / \gamma) \sqrt{2(1-\cos \theta)}$. Eqs. (30) and (31) indicate that under the Boltzmann equation approach, the average of the finitetemperature inverse relaxation time for graphene (which has a linear dispersion $E \sim k$ ) should be carried out via $\langle 1 / \tau\rangle$ $\sim \int d E E^{3}[-d f(E) / d E][1 / \tau(E)]$. This finding is in sharp contrast to the case of a conventional electron gas (which has a parabolic dispersion relation $E \sim k^{2}$ ) where the average can be done via $\langle 1 / \tau\rangle \sim \int d E E[-d f(E) / d E][1 / \tau(E)]$. We note that at present, most of the published theoretical work ${ }^{10,11}$ has used the latter average for the evaluation of the scattering time or rate in graphene.

In this study, we consider a gate-controlled graphene placed on a dielectric $\mathrm{SiO}_{2}$ wafer. The gate itself is an insulator deposited on top of the graphene sheet. The positive (negative) voltage across the gate, the graphene sheet, and the wafer layer can pull the electrons (holes) out from the $\mathrm{SiO}_{2}$ wafer and inject them into the graphene layer, while leaving behind the holes (electrons) in the substrate wafer closed to the contact. Therefore, there are always two layers of charges in the entire graphene device system: the active carriers in the graphene layer and the opposite charges in the substrate. The latter case is the main source of remoteimpurity scattering for undoped graphene samples. Moreover, in case there are molecules adsorbed on graphene, the charge transfer takes place from the molecules to the graphene sheet and the molecules can also become charged. This can result in background impurities inside the graphene layer. Hence, there are two types of impurity scattering present in the graphene system: remote impurities in the $\mathrm{SiO}_{2}$ wafer layer and background impurities in the graphene layer. When the charged impurities are located in the $\mathrm{SiO}_{2}$ substrate with a distance $d$ to the interface of the graphene layer, we can model the impurity distribution as $n_{i}(z)$ $=n_{r} \delta(z+d)$ with $n_{r}$ being the areal impurity density inside the $\mathrm{SiO}_{2}$ layer and, as a result,

$$
\left[\frac{\sigma_{0}}{\sigma_{t}^{r}(E, 0)}, \frac{\sigma_{0}}{\sigma_{q}^{r}(E, 0)}\right]=R_{1} n_{r} \int_{0}^{\pi} \frac{d \theta e^{-2 d q}}{\left(q+K_{s}\right)^{2}}\left[A_{\theta}^{t}, A_{\theta}^{q}\right],
$$

where $R_{1}=\left(2 \pi Z e^{2} / \gamma \kappa_{1}\right)^{2} / \pi$ with $\kappa_{1}$ being the dielectric constant for a system where the impurities are located in a $\mathrm{SiO}_{2}$. For background impurities located inside the graphene layer, we can model the impurity distribution as $n_{i}(z)=n_{b} \delta(z)$ with $n_{b}$ being the background-impurity density. Thus, we have

$$
\left[\frac{\sigma_{0}}{\sigma_{t}^{b}(E, 0)}, \frac{\sigma_{0}}{\sigma_{q}^{b}(E, 0)}\right]=R_{2} n_{b} \int_{0}^{\pi} \frac{d \theta}{\left(q+K_{s}\right)^{2}}\left[A_{\theta}^{t}, A_{\theta}^{q}\right],
$$

where $R_{2}=\left(2 \pi Z e^{2} / \dot{\gamma} \kappa_{2}\right)^{2} / \pi$ with $\kappa_{2}$ being the dielectric constant for a system where the impurities are located in the graphene layer. In particular, for $T \rightarrow 0$, we have $d f(E) / d E$ $\rightarrow-\delta\left(E_{F}-E\right)$ and

$$
\begin{aligned}
{\left[\frac{\sigma_{0}}{\sigma_{t}^{r}(0)}, \frac{\sigma_{0}}{\sigma_{q}^{r}(0)}\right] } & =\left[\frac{\sigma_{0}}{\sigma_{t}^{r}\left(E_{F}, 0\right)}, \frac{\sigma_{0}}{\sigma_{q}^{r}\left(E_{F}, 0\right)}\right] \\
& =R_{1} n_{r} \int_{0}^{\pi} \frac{d \theta e^{-2 d q}}{\left(q+K_{s}\right)^{2}}\left[1-\cos ^{2} \theta, 1+\cos \theta\right]
\end{aligned}
$$

and

$$
\begin{aligned}
{\left[\frac{\sigma_{0}}{\sigma_{t}^{b}(0)}, \frac{\sigma_{0}}{\sigma_{q}^{b}(0)}\right] } & =\left[\frac{\sigma_{0}}{\sigma_{t}^{b}\left(E_{F}, 0\right)}, \frac{\sigma_{0}}{\sigma_{q}^{b}\left(E_{F}, 0\right)}\right] \\
& =R_{2} n_{b} \int_{0}^{\pi} \frac{d \theta}{\left(q+K_{s}\right)^{2}}\left[1-\cos ^{2} \theta, 1+\cos \theta\right],
\end{aligned}
$$

with $q=k_{F} \sqrt{2(1-\cos \theta)}$. Consequently, the total inverse transport and quantum conductivities are, respectively,

$$
1 / \sigma_{t}(T)=1 / \sigma_{t}^{r}(T)+1 / \sigma_{t}^{b}(T),
$$

and

$$
1 / \sigma_{q}(T)=1 / \sigma_{q}^{r}(T)+1 / \sigma_{q}^{b}(T) .
$$

We note that when $K_{s} \rightarrow 0$, the transport conductivity converges over all scattering angles $0 \leq \theta \leq \pi$, whereas the quantum conductivity diverges at $\theta \rightarrow 0$. Hence, when calculating the quantum conductivity induced by impurity scattering, the effect of $c-c$ screening has to be included. When 
$K_{s}=0$ and $T \rightarrow 0$ for background-impurity scattering, we have

$$
\sigma_{t}^{b}(0)=\sigma_{0}\left(\gamma \kappa_{2} / Z e^{2}\right)^{2} /(2 \pi)\left(n_{e} / n_{b}\right)
$$

that depends linearly on $n_{e} / n_{b}$, which is similar to those obtained by taking the short-range impurity scattering. ${ }^{9-11,19}$ For the case of remote-impurity scattering at $K_{s}=0$ and $T$ $\rightarrow 0$, if the spacer distance $d$ is so small that $\sqrt{n_{e}} d \ll 1$, we have

$$
\sigma_{t}^{r}(0) \simeq \sigma_{0}\left(\gamma \kappa_{1} / Z e^{2}\right)^{2} /(2 \pi)\left(n_{e} / n_{r}\right),
$$

which depends also linearly on $n_{e} / n_{r}$.

The results obtained from this work suggest that under the Boltzmann equation approach, if $1 / \sigma(E, 0)$ is the inverse conductivity at $T \rightarrow 0$, the finite-temperature conductivity can be evaluated generally through using Eq. (31). This is in contrast to a conventional electron gas with a parabolic dispersion where

$$
\frac{1}{\sigma(T)}=\int_{0}^{\infty} d E \frac{E}{\sigma(E, 0)} \frac{d f(E)}{d E} / \int_{0}^{\infty} d E E \frac{d f(E)}{d E} .
$$

We find that only for background-impurity scattering in the absence of electron-electron screening (i.e., $K_{s}=0$ ), the transport conductivity can be calculated through

$$
\frac{1}{\sigma_{t}^{b}(T)}=-\frac{1}{E_{F}} \int_{0}^{\infty} d E \frac{E}{\sigma_{t}^{b}(E)} \frac{d f(E)}{d E}
$$

because $\sigma(E, 0) \sim q^{2}$ and $q \sim E$. For the case of $K_{s} \neq 0$ or for remote-impurity scattering, we have to use Eq. (31) to obtain the finite-temperature conductivity from the zerotemperature one. For the case of a high density graphene sample at relatively low-temperatures so that $E_{F} \gg k_{B} T$ and $\sigma_{l}(E, 0) \rightarrow \sigma_{l}\left(E_{F}, 0\right)=\sigma_{l}(0)$, Eq. (31) takes the asymptotic expression

$$
\frac{1}{\sigma_{l}(T)} \simeq \frac{1}{\sigma_{l}(0)}\left[1+\left(\pi k_{B} T / E_{F}\right)^{2} / 2\right]
$$

\section{NUMERICAL RESULTS AND DISCUSSIONS}

In the numerical calculations, we take the band parameter for graphene $\gamma=\hbar v_{F}$ with $v_{F}=10^{8} \mathrm{~cm} / \mathrm{s}$. We take the dielectric constants for bare graphene layer and bare $\mathrm{SiO}_{2}$ wafer to be $\kappa_{\text {graphene }}=4.5$ and $\kappa_{\mathrm{SiO}_{2}}=4.0$, respectively. Because the graphene device is an air-graphene-substrate system with different dielectric constants in different layers, there is a mismatch of the dielectric constants at the interfaces. For the case where the charged impurities are located in the $\mathrm{SiO}_{2}$ substrate (i.e., for remote impurities), we note that normally $n_{r} \sim n_{e} \gg n_{b}$. As a result, the graphene sheet is polarized and there are polarized mirror image charges in graphene. Taking this effect into consideration, the effective dielectric constant for the remote impurities and polarized mirror image charges can be evaluated using image charge method. For a twodimensional plane surface, the effective dielectric constant for remote impurities in $\mathrm{SiO}_{2}$ substrate is given simply as $\kappa_{1}=\left[\kappa_{\mathrm{sio}_{2}}+\kappa_{\text {graphene }}\right] / 2=4.25$. For charged impurities (i.e.,

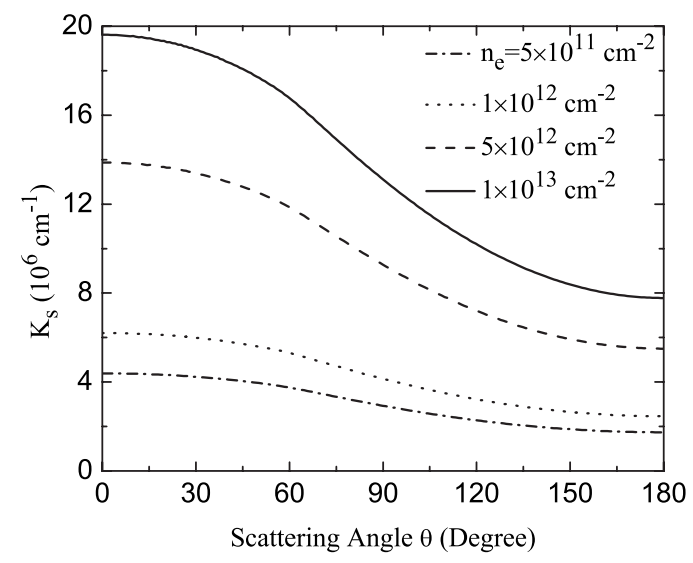

FIG. 1. The inverse screening length as a function of scattering angle $\theta$ for $T \rightarrow 0$ at different electron densities $n_{e}$, as indicated. Here the change of the electron wave vector is $q$ $=\sqrt{2 \pi n_{e}(1-\cos \theta)}$ and $q=\left[0,2 k_{F}\right]$ when $\theta=[0, \pi]$ with $k_{F}=\sqrt{\pi n_{e}}$ being the Fermi wave vector.

background impurities) and electrons located within the graphene sheet, it is not so easy to evaluate the corresponding effective dielectric constant because the graphene layer is placed between the $\mathrm{SiO}_{2}$ substrate and air. However, we note that the graphene layer is atomic layer thin, which is much smaller than the substrate thickness and air medium. Together with the fact that normally the background-impurity concentration is $n_{b} \ll n_{r} \sim n_{e}$, the effective dielectric constant for the background impurities and conducting electrons is then determined by the $\mathrm{SiO}_{2}$ substrate and air, which relates to $r_{s}$, the effective fine structure constant (or coupling constant). For high carrier-density samples, $r_{s} \ll 1$ and the effective dielectric constant for background impurities and electrons in graphene is given as $\kappa_{2}=\left[\kappa_{0}+\kappa_{\text {sio }_{2}}\right]\left(1+\pi r_{s} / 2\right) / 2$ $\simeq 2.5$ with $\kappa_{0}=1$ being the dielectric constant of the air. $\kappa_{2}$ can be used to calculate the quantum and transport conductivities induced by background-impurity scattering, and to evaluate the screening length induced by the electronelectron interaction. Moreover, in the calculations, the typical electron density is taken to be $n_{e} \sim 10^{12} \mathrm{~cm}^{-2}$ and the charge number of an impurity to be $Z=1$. When $n_{e}$ $=10^{12} \mathrm{~cm}^{-2}, E_{F}=\gamma \sqrt{\pi n_{e}} \sim 1354 \mathrm{~K}$ is much larger than room temperature $T=300 \mathrm{~K}$. Thus, we can use Eq. (38) to evaluate the dependence of the conductivity on temperature up to room temperature.

In Fig. 1, we plot the inverse RPA screening length $K_{s}$ given by Eq. (18) as a function of the scattering angle $\theta$ for different electron densities $n_{e}$. Noting that the change of the electron wave vector is $q=\sqrt{2 \pi n_{e}(1-\cos \theta)}$, we find that $K_{s}$ increases with $n_{e}$ for a fixed $\theta$ and decreases with increasing $\theta$ at a fixed $n_{e}$. The strongest effect of electron-electron screening is achieved at $\theta \rightarrow 0$ or $q \rightarrow 0$. When $\theta=[0, \pi], q$ $=\left[0,2 k_{F}\right]$ with $k_{F}=\sqrt{\pi n_{e}}$ being the Fermi wave vector and $K_{s}$ is reduced by a factor of about 2. In Fig. 2, we show the temperature dependence of the RPA screening length given by Eq. (17). We find that at a fixed $n_{e}, K_{s}$ decreases with increasing temperature and the strongest effect of electronelectron screening is achieved at $T \rightarrow 0$. At finite temperature, $K_{s}$ has a similar dependence on $\theta$ as that at $T \rightarrow 0$. The results 


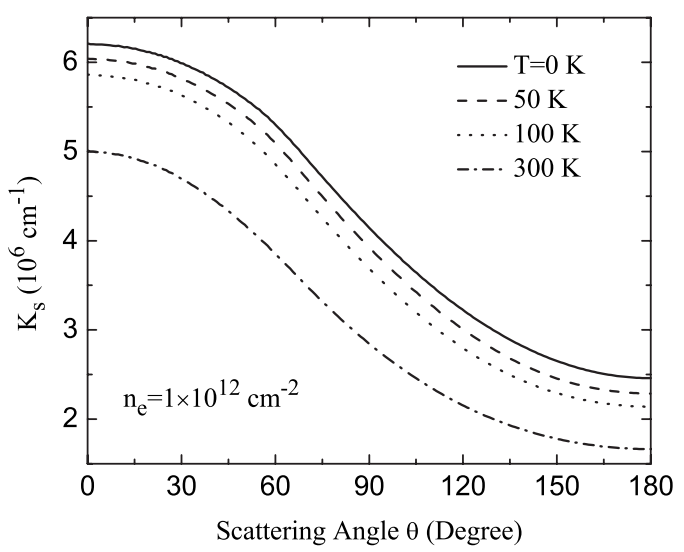

FIG. 2. The inverse screening length as a function of scattering angle $\theta$ for a fixed electron density $n_{e}$ at different temperatures $T$, as indicated.

obtained from our numerical calculations show that for a typical graphene device with an electron density $n_{e}$ $\sim 10^{12} \mathrm{~cm}^{-2}$, the RPA inverse screening length is $K_{s}$ $\sim 10^{6} \mathrm{~cm}^{-1}$ over a wide temperature range up to room temperature.

In Fig. 3, we plot the quantum and transport conductivities at $T \rightarrow 0$ [i.e., $\sigma_{t}(0)$ and $\left.\sigma_{q}(0)\right]$ as a function of electron density $\left(n_{e}\right)$ for the fixed background-impurity density $\left(n_{b}\right)$ and spacer distance $(d)$ for different remote-impurity concentrations $\left(n_{r}\right)$. We compare the theoretical results with those obtained experimentally (symbols). ${ }^{10}$ As can be seen, when taking $\quad n_{b}=1.5 \times 10^{11} \mathrm{~cm}^{-2}, \quad 2.5 \times 10^{11} \mathrm{~cm}^{-2}, \quad 3.5$ $\times 10^{11} \mathrm{~cm}^{-2}$, and $d=2 \AA$, the present theoretical results agree well with the experimental data and with those obtained theoretically in Ref. 9 for $n_{r}=10^{12}, 2 \times 10^{-2}$, and 3 $\times 10^{12} \mathrm{~cm}^{-2}$. It should be noted that in Ref. 9, the small $q$ approximation was used to calculate the inverse screening

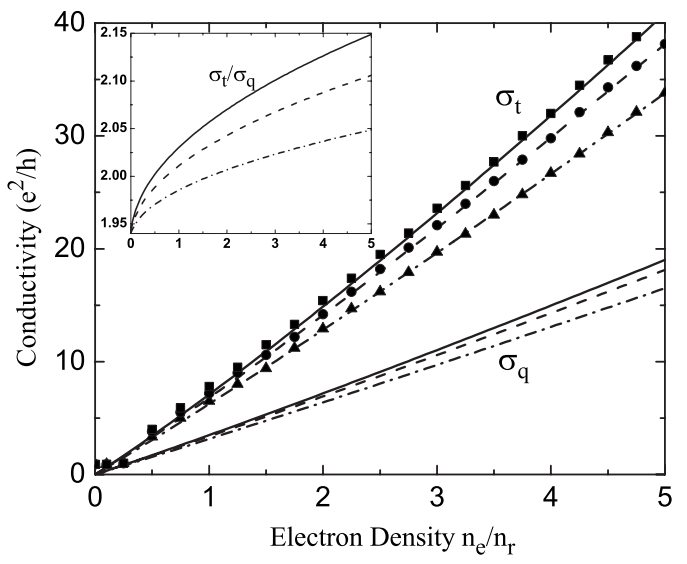

FIG. 3. Transport $\sigma_{t}$ and quantum $\sigma_{q}$ conductivities as a function of electron density $n_{e}$ at a fixed spacer distance $d=2 \AA$ for different remote- and background-impurity concentrations $\left[n_{r}\right.$ $=10^{12}$ and $n_{b}=1.5 \times 10^{11} \mathrm{~cm}^{-2}$ (solid curves), $n_{r}=2 \times 10^{12}$ and $n_{b}$ $=2.5 \times 10^{11} \mathrm{~cm}^{-2}$ (dashed curves), and $n_{r}=3 \times 10^{12}$ and $n_{b}=3.5$ $\times 10^{11} \mathrm{~cm}^{-2}$ (dashed and dotted curves)]. The results are obtained for $T \rightarrow 0$. The symbols are the experimental results from Ref. 10 and the inset shows the ratio between the transport and quantum conductivities, $\sigma_{t}(0) / \sigma_{q}(0)$.

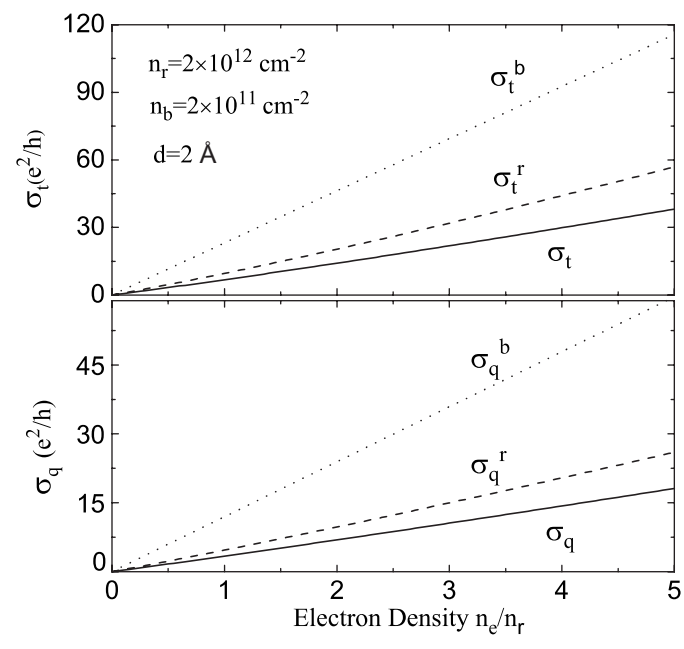

FIG. 4. Contributions from remote- and background-impurity scatterings to the total transport (upper panel) and quantum (lower panel) conductivities as a function of electron density for $T \rightarrow 0$.

length $K_{s} \sim q_{s} \sim k_{F} \sim \sqrt{n_{e}}$ [see Eq. (19)]. As a result, $\sigma_{t}(0)$ $\sim n_{e}$ was observed in Ref. 9 . In contrast, the results shown in Fig. 3 are obtained by using a more general expression for $K_{s}$ [Eq. (17)] without the usage of the small $q$ approximation. The linear dependence of $\sigma_{t}(0)$ on $n_{e}$ can still be observed. The reason behind this is that the strongest effect of electron-electron screening is achieved at small scattering angles or $q \rightarrow 0$, as shown in Fig. 1. With increasing $q, K_{s}$ decreases (see Fig. 1) and, therefore, the screening effect becomes weaker for electron-impurity scattering. Our results demonstrate that for effective carrier-impurity scattering in graphene, the small $q$ approximation for the RPA screening length can result in a fairly good approximation to the calculation of the transport and quantum conductivities. We find that in graphene, not only does the transport conductivity depend linearly on electron density but so does the quantum conductivity $\left[\sigma_{q}(0)\right]$. This needs to be verified experimentally. In the inset of Fig. 3, we show the ratio $\sigma_{t}(0) / \sigma_{q}(0)$ between the transport and quantum conductivities. Because the transport conductivity is induced by electronic scattering at all angles whereas the quantum conductivity resulted from only small-angle scattering events, $\sigma_{t}(0)$ is normally larger than $\sigma_{q}(0)$. Similar to a conventional two-dimensional electron gas, $\sigma_{t}(0) / \sigma_{q}(0)$ is about a factor of 2 and increases with increasing electron density. ${ }^{13}$

In Fig. 4, we show the contributions from remote- and background-impurity scattering to the transport (upper panel) and quantum (lower panel) conductivities. The results are obtained in conjunction with those shown in Fig. 3. We find that for high quality graphene samples in which the background-impurity concentrations are low, the quantum and transport conductivities are mainly determined by electronic scattering with remote impurities. Another reason for a relatively weaker background-impurity scattering is that for a graphene sheet on $\mathrm{SiO}_{2}$ wafer: the effective dielectric constant for background impurities $\kappa_{2} \sim 2.5$ is smaller than that for remote impurities in the $\mathrm{SiO}_{2}$ wafer $\kappa_{1} \sim 4.25$, owing to the effect of the dielectric constant mismatch in the device system. From a practical point of view, it is inconvenient and 


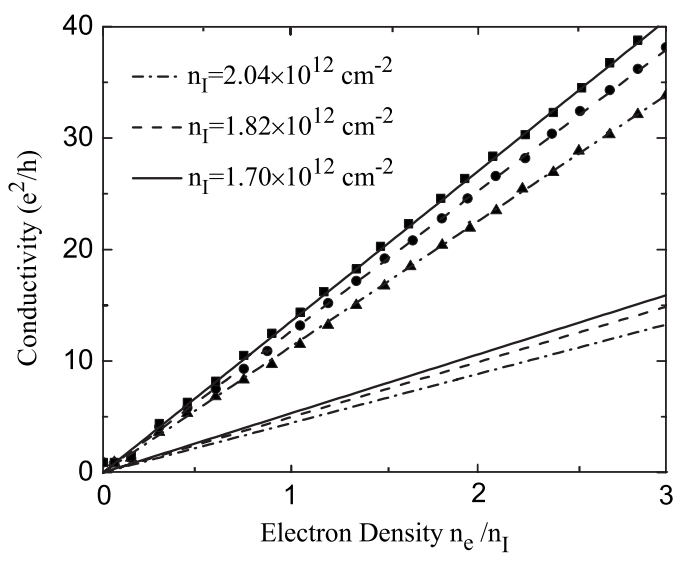

FIG. 5. Transport and quantum conductivities as a function of electron density $n_{e} / n_{I}$ for different effective impurity concentrations $n_{I}$, as indicated. The results are obtained from Eq. (39) for $T \rightarrow 0$ and the experimental results (Ref. 10) are shown for comparison.

unnecessary to use three sample parameters (namely $n_{r}, n_{b}$, and $d$ used in Fig. 1 in Ref. 9) to fit the experimental data, which shows a linear dependence. On the basis that: (i) the background impurities contribute weakly to the quantum and transport conductivities (see Fig. 4), (ii) for remote impurities in a typical graphene sample with $n_{e} \sim 10^{12} \mathrm{~cm}^{-2}$, $d \sqrt{n_{e}} \ll 1$ when $d \sim 2 \AA$, and (iii) the strongest effect of electron-electron screening on electron-impurity scattering occurs at $q \rightarrow 0$ (see Fig. 1), we may assume that the effective impurities with an effective concentration $n_{I}$ are located in the $\mathrm{SiO}_{2}$ substrate but are very close to the interface between the graphene sheet and the substrate (i.e., $d \simeq 0$ ), and we can use $q \rightarrow 0$ approximation for the inverse screening length $K_{s}$. Thus, the quantum and transport conductivities can be evaluated simply through

$$
\begin{aligned}
{\left[\sigma_{t}(0), \sigma_{q}(0)\right] } & =\left(\frac{\gamma \kappa_{1}}{2 e^{2}}\right)^{2} \frac{\sigma_{0} n_{e}}{n_{I}} / \int_{0}^{1} d x \frac{\sqrt{1-x^{2}}\left[2 x^{2}, 1\right]}{\left(x+2 e^{2} / \gamma \kappa_{2}\right)^{2}} \\
& \simeq \frac{e^{2} \kappa_{1}^{2}}{h} \frac{n_{e}}{n_{I}}[0.768,0.301] .
\end{aligned}
$$

Using Eq. (39), we can use only one fitting parameter $n_{I}$ or even $n_{e} / n_{I}$ to compare with the experimental results of both the quantum and transport conductivities. By taking $n_{I}$ $\sim 2.04 \times 10^{12}, 1.82 \times 10^{12}$, and $1.70 \times 10^{12} \mathrm{~cm}^{-2}$, we can also reach a good agreement with the experimental results for the transport conductivity (see Fig. 5).

In Fig. 6, we show the dependence of the transport and quantum conductivities on temperature. Here we use Eq. (39) to evaluate the conductivities at $T \rightarrow 0$ and use Eq. (38) to count for the temperature effect. For a typical graphene device with an electron density $n_{e} \sim 10^{12} \mathrm{~cm}^{-2}, \quad E_{F}$ $\sim 1354 \mathrm{~K}$ is about four times larger than room temperature. Therefore, Eq. (38) is a good approximation for the temperature dependence of the electron-impurity scattering in graphene. We find that the quantum and transport conductivities induced by impurity scattering depend weakly on temperature up to room temperature, and $\sigma_{t}(T)$ and $\sigma_{q}(T)$ decrease slightly with increasing $T$. From Eq. (38), we see that

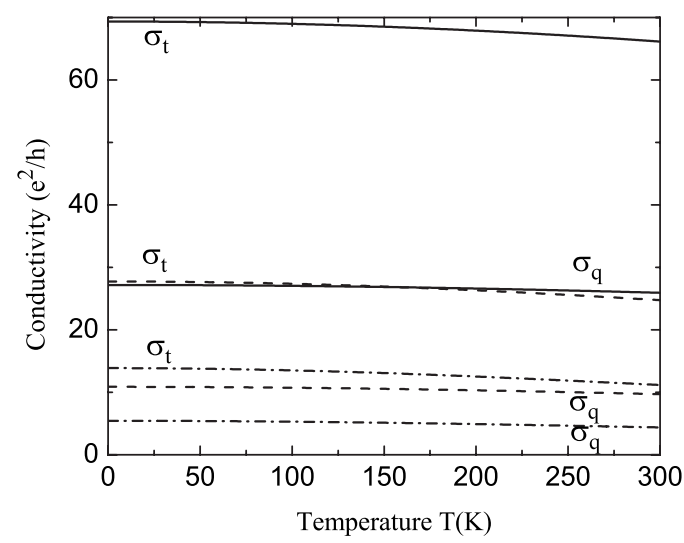

FIG. 6. Temperature dependence of the quantum and transport conductivities at a fixed impurity concentration $n_{I}=10^{12} \mathrm{~cm}^{-2}$ for different electron densities $n_{e}=10^{12}$ (dotted-dashed curves), 2 $\times 10^{12}$ (dashed curves), and $5 \times 10^{12} \mathrm{~cm}^{-2}$ (solid curves). The results are obtained using Eqs. (38) and (39).

the larger the electron density is, the weaker the temperature dependence is for graphene. This is in line with very recent experimental findings. ${ }^{20}$

\section{CONCLUSIONS}

In this paper, we developed a simple and transparent theory to study the electronic transport properties of monolayer graphene. The proposed theoretical approach is based on the momentum-balance equation derived from the semiclassical Boltzmann equation. Including the electronimpurity scattering in typical graphene devices where the electron-electron screening is counted by the usual RPA approach, we have studied the dependence of the quantum and transport conductivities on electron density, remote- and background-impurity concentrations, temperature, etc. Furthermore, we have applied the published experimental results to examine the validity of the proposed model calculations. The main conclusions obtained from this study are summarized as follows.

Under the Boltzmann equation approach, the average of the finite-temperature relaxation time or scattering rate for Dirac particles in graphene should be carried out in general through $\langle 1 / \tau\rangle \sim \int d E E^{3}[-d f(E) / d E][1 / \tau(E)]$, in contrast to $\langle 1 / \tau\rangle \sim \int d E E[-d f(E) / d E][1 / \tau(E)]$ used previously in Refs. 9 and 11. The latter average holds only for an electron gas system with a parabolic dispersion $E \sim k^{2}$. For graphene, due to its linear dispersion relation $E \sim k$, the former one is more correct in counting the energy average. Although different energy averages on electronic scattering rate are used in the present study, we still observe a linear dependence of the quantum and transport conductivities on the electron density.

We have used a RPA screening length without the small $q$ approximation to calculate the quantum and transport conductivities. We find that the inclusion of a more general expression for the inverse RPA screening length does not change the linear dependence of the conductivities on electron density in graphene. The main reason behind this feature is that the strongest electron-electron screening effect on electron impurity is achieved for $q \rightarrow 0$. 
On the basis of the results obtained from this study, we have proposed a very simple analytical formula [given by Eq. (39)] to calculate the quantum and transport conductivities in graphene. Using this formula, we can compare our theoretical results with the experimental results using only one fitting parameter (i.e., the effective impurity concentration $n_{I}$ or the ratio $\left.n_{e} / n_{I}\right)$.

We have examined the temperature dependence of the quantum and transport conductivities in typical graphene samples. We find that when the electron density is about $10^{12} \mathrm{~cm}^{-2}$, the Fermi energy (or Fermi temperature) of graphene is about four times larger than room temperature. Thus, the conductivities induced by impurity scattering depend rather weakly on temperature up to room temperature.

We studied the dependence of quantum conductivity on electron density, different impurity scattering channels, and temperature. We find that similar to a conventional twodimensional electron gas, the quantum conductivity induced by small angle scattering is about twice smaller than the transport conductivity. The quantum conductivity has a simi- lar dependence on the electron density and temperature as the transport one.

The transport conductivity in monolayer graphene has been well studied both experimentally and theoretically. The quantum conductivity (or quantum lifetime) for a Dirac quasiparticle in graphene has been overlooked up to now. From the fact that the $\mathrm{SdH}$ oscillations along with the quantum Hall effect can be observed at relatively high temperatures in graphene, we believe that the quantum conductivity can be easily determined experimentally. Hence, we hope the theoretical findings and predictions in this paper, especially those regarding quantum conductivity, can be verified experimentally.

\section{ACKNOWLEDGMENTS}

This work was supported by the Chinese Academy of Sciences, Ministry of Education of China, and by the Flemish Science Foundation (FWO-Vl). *wen105@rsphysse.anu.edu.au

†Francois.Peeters@ua.ac.be

${ }^{1}$ K. S. Novoselov, A. K. Geim, S. V. Morozov, D. Jiang, M. I. Katsnelson, I. V. Grigoreva, S. V. Dubonos, and A. A. Firsov, Nature (London) 438, 197 (2005); K. S. Novoselov, A. K. Geim, S. V. Morozov, D. Jiang, Y. Zhang, S. V. Dubonos, I. V. Grigoreva, and A. A. Firsov, Science 306, 666 (2004).

${ }^{2}$ Yuanbo Zhang, Yan-Wen Tan, Horst L. Stormer, and Philip Kim, Nature (London) 438, 201 (2005).

${ }^{3}$ N. Tombros, Csaba Jozsa, Mihaita Popinciuc, Harry T. Jonkman, and Bart J. Van Wees, Nature (London) 448, 571 (2007).

${ }^{4}$ See, e.g., the special issue on graphene, edited by S. Das Sarma, A. K. Geim, P. Kim, and A. H. MacDonald [Solid State Commun. 143, 1-123 (2007)].

${ }^{5}$ See, e.g., J. R. Williams, L. C. DiCarlo, and C. M. Marcus, Science 317, 638 (2007).

${ }^{6}$ See, e.g., B. Özyilmaz, P. Jarillo-Herrero, D. Efetov, D. A. Abanin, L. S. Levitov, and P. Kim, Phys. Rev. Lett. 99, 166804 (2007).

${ }^{7}$ See, e.g., A. K. Geim and K. S. Novoselov, Nat. Mater. 6, 183 (2007).

${ }^{8}$ Y.-W. Tan, Y. Zhang, K. Bolotin, Y. Zhao, S. Adam, E. H. Hwang, S. Das Sarma, H. L. Stormer, and P. Kim, Phys. Rev. Lett. 99, 246803 (2007); S. V. Morozov, K. S. Novoselov, M. I.
Katsnelson, F. Schedin, D. Elias, J. A. Jaszczak, and A. K. Geim, ibid. 100, 016602 (2008).

${ }^{9}$ K. Nomura and A. H. MacDonald, Phys. Rev. Lett. 98, 076602 (2007); 96, 256602 (2006).

${ }^{10}$ E. H. Hwang, S. Adam, and S. Das Sarma, Phys. Rev. Lett. 98, 186806 (2007).

${ }^{11}$ F. T. Vasko and V. Ryzhii, Phys. Rev. B 76, 233404 (2007); T. Stauber, N. M. R. Peres, and F. Guinea, ibid. 76, 205423 (2007).

${ }^{12}$ T. Stauber, N. M. R. Peres, and F. Guinea, Phys. Rev. B 76, 205423 (2007).

${ }^{13}$ See, e.g., R. Fletcher, J. J. Harris, C. T. Foxon, and R. Stoner, Phys. Rev. B 45, 6659 (1992).

${ }^{14}$ J. W. McClure, Phys. Rev. 104, 666 (1956).

${ }^{15}$ See, e.g., R. G. Mattuck, A Guide to Feynman Diagrams in the Many-Body Problem (McGraw-Hill, New York, 1976).

${ }^{16}$ W. Xu, F. M. Peeters, and J. T. Devreese, Phys. Rev. B 43, 14134 (1991).

${ }^{17}$ W. Xu and C. Zhang, Phys. Rev. B 55, 5259 (1997).

${ }^{18}$ W. Xu, Phys. Rev. B 71, 245304 (2005).

${ }^{19}$ M. Auslender and M. I. Katsnelson, Phys. Rev. B 76, 235425 (2007).

${ }^{20}$ J. H. Chen, C. Jang, S. Xiao, M. Ishigami, and M. S. Fuhrer, Nat. Nanotechnol. 3, 206 (2008). 